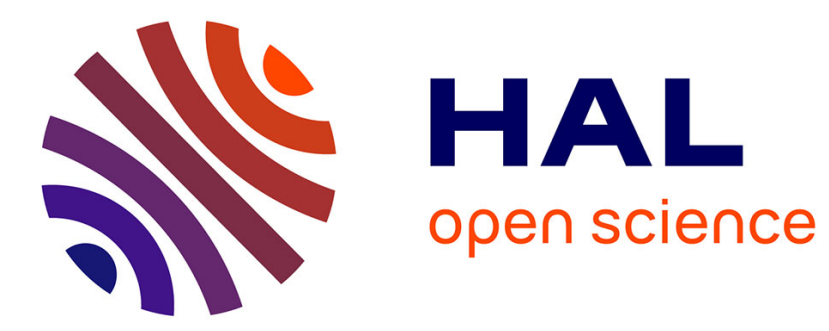

\title{
Ajout de détails dynamiques à une animation temps-réel de personnage
}

\author{
Caroline Larboulette, Marie-Paule Cani, Bruno Arnaldi
}

\section{To cite this version:}

Caroline Larboulette, Marie-Paule Cani, Bruno Arnaldi. Ajout de détails dynamiques à une animation temps-réel de personnage. Revue des Sciences et Technologies de l'Information - Série TSI : Technique et Science Informatiques, 2006, 25 (6), pp.759-789. inria-00402593

\section{HAL Id: inria-00402593 https://hal.inria.fr/inria-00402593}

Submitted on 20 May 2011

HAL is a multi-disciplinary open access archive for the deposit and dissemination of scientific research documents, whether they are published or not. The documents may come from teaching and research institutions in France or abroad, or from public or private research centers.
L'archive ouverte pluridisciplinaire HAL, est destinée au dépôt et à la diffusion de documents scientifiques de niveau recherche, publiés ou non, émanant des établissements d'enseignement et de recherche français ou étrangers, des laboratoires publics ou privés. 


\title{
Ajout de détails dynamiques à une animation temps-réel de personnage
}

\author{
Caroline Larboulette, ${ }^{* * * * * * *}$-Marie-Paule Cani* - Bruno Arnaldi** \\ * Laboratoire GRAVIR ${ }^{1}, 655$ avenue de l'Europe, 38334 Saint Ismier Cedex \\ Marie-Paule.Cani@imag.fr \\ ** Laboratoire IRISA ${ }^{2}$, Campus de Beaulieu, 35042 Rennes Cedex \\ Bruno.Arnaldi@irisa.fr \\ *** Actuellement à TU Wien, Favoritenstrasse 9-11, A 1040 Wien \\ Caroline.Larboulette@cg.tuwien.ac.at
}

\begin{abstract}
RÉSUMÉ. L'animation temps-réel de personnages repose généralement sur une technique d'habillage, appelée skinning en anglais, permettant à la peau et aux vêtements de suivre les mouvements du squelette. Cette technique n'engendre malheureusement aucun des effets dynamiques - vibrations des tissus non-rigides, plissement variable de la peau et des vêtements - pourtant cruciaux pour le réalisme. Dans cet article, nous présentons deux techniques complémentaires pour ajouter ces effets de manière intuitive, tout en limitant au maximum les sur-coûts lors $d u$ calcul de l'animation. Combinables entre eux et rapides à mettre en place, nos outils améliorent le réalisme visuel du mouvement sans nécessiter de modification de la séquence d'animation de départ, de précalcul mécanique ni de modification des modèles. Nous illustrons nos résultats sur des animations variées de personnages et d'animaux.
\end{abstract}

ABSTRACT. Real-time animation of characters often relies on a geometric technique called skinning which enables the skin and clothes to follow the skeleton motion. However, dynamic effects - non-rigid tissues vibrations, dynamic wrinkles of skin and clothes - can not be generated by $i t$. However such deformations are of primary importance for the realism of the generated animation. In this paper, we propose two intuitive methods which address those specific problems with little increase in computational cost of the animation. Our tools can be combined together on an existing animation sequence to enhance its realism, with no need to change the original animation, no mechanics precomputation and no modification of the model. We illustrate our results with several characters and animals animations.

MOTS-CLÉS : modélisation, animation procédurale, temps-réel, plis, longueur constante

KEYWORDS: modelisation, procedural animation, real-time, wrinkles, constant length

1. GRAVIR est un laboratoire composé du CNRS, de l'INP de Grenoble, de l'INRIA et de l'Université Joseph Fourier.

2. IRISA est un laboratoire composé du CNRS, de l'INRIA, de l'INSA de Rennes et de l'Université de Rennes 1.

L'objet - 6/2006, pages 1 à 29 


\section{Introduction}

Les animations de personnages $3 D$ que l'on rencontre au cinéma, à la télévision ou bien dans les jeux vidéos sont de plus en plus réalistes, sans être totalement satisfaisantes. Pour des raisons d'efforts de modélisation trop importants, ou de coûts de calculs devant être réduits (temps-réel pour le jeu vidéo par exemple), les modèles et les animations nécessitent d'être simplifiés. Cette simplification porte en priorité sur les détails comme les effets dynamiques des masses musculaires ou graisseuses ou bien les plis non statiques de la peau ou des vêtements.

L'objectif de cet article est de proposer des techniques géométriques permettant de rajouter ces détails. Elles doivent être peu coûteuses en temps de calcul, simples à utiliser, ajoutables comme des couches supplémentaires du modèle et utilisables sur des animations existantes. En effet, nous avons souhaité bénéficier des avantages d'une méthode bien connue, le skinning [LEW 00, BLO 02]. C'est une technique géométrique qui exprime la position d'un sommet du maillage déformé comme la somme pondérée de ses positions rigides dans tous les repères du squelette dont il dépend. Bien que cette technique dans sa version originale souffre de quelques inconvénients, notamment au niveau des articulations, un certain nombre de travaux récents [BLO 02, WAN 02, KRY 02, MOH 03] apportent des solutions satisfaisantes en spécifiant des repères ou des poids supplémentaires ainsi que des dépendances aux repères adaptées. Cependant, ces solutions ne permettent pas de résoudre les problèmes que nous nous sommes posés.

C'est pourquoi nous avons développé deux techniques que nous présentons en détail dans cet article : un skinning dynamique permettant d'ajouter des effets d'inertie des tissus sous-cutanés et un outil de création de plis géométriques. La seconde technique a déjà fait l'objet d'une publication internationale [LAR 04b]. Par contre, le skinning dynamique (détaillé dans [LAR 04a]) simulant des effets dynamiques de la chair ainsi que sa combinaison avec notre outil de plis sont de nouvelles contributions.

\subsection{Travaux antérieurs}

\subsubsection{Effets dynamiques sur les muscles et les tissus}

La première tentative pour ajouter des effets dynamiques à une technique géométrique de déformation des muscles a été apportée par Chadwick [CHA 89]. Sa technique, dont les déformations cinématiques sont simulées par une double grille de FFDs, consiste à ajouter des masses aux nœuds de la grille et des ressorts entre ces masses. Certaines masses sont fixées au squelette ce qui a pour conséquence d'étirer les ressorts et d'obtenir un comportement dynamique de la grille de points de contrôle. L'idée d'utiliser des ressorts a été reprise par Turner [TUR 93] puis Wilhelms [WIL 97a, WIL 97b] pour calculer le comportement d'une couche de peau élastique attachée aux muscles, os ou parties graisseuses sous-cutanées par l'intermédiaire de ressorts. La peau peut donc s'étirer, glisser sur les muscles, mais ces tech- 
niques basées sur l'anatomie requièrent malheureusement des efforts de modélisation très importants de la part de l'utilisateur.

Des techniques basées sur les lois physiques de l'élasticité ont été utilisées avec succès dans différents travaux. Ainsi, Capell [CAP 02] anime des tissus déformables en plongeant l'objet dans une grille de contrôle facilement paramétrable qui peut se raffiner par subdivision et sur laquelle une base hiérarchique est définie. Chaque élément de la grille est alors un élément fini et ces sont les éléments les plus fins qui sont actifs dans les zones où plus de détails sont nécessaires. Müller [M“02] simule des grandes déformations en temps-réel en utilisant une matrice linéaire précalculée qui est ensuite transformée par un champ de tenseurs qui décrit les rotations locales du matériau déformé. Enfin, James [JAM 02] précalcule les modes de vibrations d'un maillage local d'éléments finis et les combines en temps-réel en utilisant une implémentation hardware de l'analyse modale [PEN 89]. Les inconvénient majeurs de ces techniques est qu'il est nécessaire d'avoir une représentation volumique du modèle que l'on déforme, et que les paramètres de la simulation physique sont souvent difficiles à régler pour un utilisateur non expert.

\subsubsection{Plis de la peau et de vêtements}

Les premiers travaux concernant la création de bosses ou de plis apparaissent dès 1978 avec la technique du bump mapping [BLI 78] qui consiste à modifier les normales des sommets du maillage avant les calculs d'éclairage pour donner une impression visuelle de plis. Cette idée a été largement reprise depuis, particulièrement en animation faciale [PAS 01, BOI 00]. Les résultats sont visuellement satisfaisants mais étant donné que la géométrie des objets n'est pas déformée, leurs silhouettes sont visuellement incorrectes ce qui est gênant pour les vues rapprochées. De plus il n'est pas possible d'utiliser un algorithme de détection de collision efficace dans les zones plissées.

Le premier problème peut être résolu par l'utilisation de displacement mapping au lieu du bump au moment du rendu. Par exemple, Volino [VOL 99] anime des plis de vêtements en modulant l'amplitude d'un schéma de plis dessiné par un infographiste, en utilisant des contraintes physiques sur chaque triangle d'un maillage grossier et en raffinant le maillage si nécessaire. Ce travail a ensuite été étendu dans [HAD 99] pour simuler de jolis plis de vêtements. Cependant, comme précisé par Kono [KON 03], les paramètres physiques sont difficiles à régler et la qualité des résultats dépend essentiellement du schéma de plis dessiné par l'utilisateur. Bando [BAN 02] utilise une interface plus intuitive pour créer la carte des déplacements mais l'utilisateur doit spécifier les rides une à une sur une projection $2 D$ du maillage. De plus, un pré-calcul relativement coûteux est nécessaire pour calculer un maillage ayant les bonnes propriétés pour que l'algorithme soit utilisable. D'autres travaux calculent la carte de déplacements à partir de modèles physiques plus complexes [WU 94, WU 96, WU 99, BOI 00]. Cependant, dans tous les cas, la géométrie du maillage reste indéformée en dehors de la carte graphique au moment du rendu final, ce qui empêche l'utilisation d'algorithmes en post-traitement comme la détection de collisions. 
D'autres techniques déforment effectivement le maillage géométrique. En animation faciale, Viaud [VIA 92] utilise un masque de rides de référence qui consiste en une surface spline dont les isolignes sont alignées avec les rides potentielles. Combaz [COM 02] génère des plis complexes en simulant les déformations statiques d'un maillage d'éléments finis, déformé par un processus de croissance interne. L'utilisateur dispose d'une interface intuitive pour peindre les directions principales des rides et leur fréquence mais la technique n'est pas temps-réel et elle ne convient pas à la génération de plis à surface constante en réponse à une animation. En effet, notre objectif est de simuler une surface qui plisse pour résister à la compression et non des plis créés par un processus d'expansion.

D'autres modèles simulent directement la déformation d'un volume ou d'une surface par des masses-ressorts ou des éléments finis, et les plis sont une conséquence de la simulation. Par exemple, les travaux récents de Choi et Ko [CHO 02] produisent des plis très réalistes sur des vêtements de personnages, dans le creux du genou en particulier et le travail de Baraff et al. [BAR 03] s'intéresse au problème des autocollisions du vêtement et produit des plis très naturels. Les principaux inconvénients de ces méthodes physiques sont qu'elles ne sont pas temps-réel et le contrôle des plis se fait uniquement à travers des paramètres physiques comme la raideur du tissu. Il est donc très difficile de choisir l'emplacement et la forme des plis.

Puisque les rides dynamiques sont dues à des contraintes de conservation de longueur inhérentes aux tissus physiques, elles peuvent logiquement être générées à partir de ces contraintes plutôt que par une simulation ou la création détaillée par un artiste. Sauvage [SAU 04] et al. ont proposé un modèle de courbe multirésolution qui préserve sa longueur pendant la manipulation en ondulant à une échelle prédéfinie. Leur solution repose sur un processus d'optimisation global qui résout les contraintes sur la longueur de la courbe limite. Cette méthode n'est malheureusement pas temps-réel et ne s'applique pas directement à un maillage.

\subsection{Méthodologie générale}

Les deux outils que nous proposons dans cet article ont été développés afin d'améliorer des animations classiques par skinning. Le modèle de personnage est constitué de deux couches : le squelette interne et la peau. La peau est un maillage triangulaire qui décrit l'enveloppe $3 D$ du personnage pour lequel les déformations statiques sont obtenues par skinning [ali]. Le squelette est composé d'une hiérarchie de repères qui permettent d'animer la peau. Dans nos animations, ils sont placés aux articulations idéales, à la main. La racine est traditionnellement choisie au niveau du bassin. Notre premier outil, appelé skinning dynamique agit comme une couche supplémentaire du modèle. L'utilisation d'un modèle physique simplifié nous permet d'ajouter des effets physiques au sein d'un système de déformations purement géométrique. La localisation des effets recherchés nous conduit à l'introduction de la notion d'élément de chair dynamique. Le modèle final de personnage est obtenu en plaçant différents éléments de chair dynamique sur la géométrie. Ces éléments vibrent pendant le mouvement 
et permettent de modifier la géométrie de la peau obtenue par smooth skinning juste avant le rendu de la scène. C'est donc une méthode de skinning classique (ou toute autre méthode équivalente) qui gère les problèmes de raccords aux articulations et la forme statique de l'objet déformé.

De la même façon, notre outil de génération de plis intervient comme une couche supplémentaire, qui s'ajoute au skinning dynamique s'il existe, au skinning classique sinon. Différents outils sont placés sur la surface du modèle. Pendant l'animation, le maillage est déformé par les skinnings successifs. Le maillage ainsi déformé sert d'entrée aux outils de plis qui déforment alors le maillage à leur tour. Il est tout à fait envisageable de rajouter des couches supplémentaires, avant, entre ou après nos outils, puisque ceux-ci prennent en entrée un maillage polygonal pour générer un maillage polygonal déformé.

Le reste de l'article s'organise de la façon suivante : la section 2 présente notre premier outil géométrique dédié à l'ajout d'effets dynamiques. La section 3 détaille le fonctionnement de notre outil de création et d'animation de plis. Ensuite, la section 4 nous montre des résultats variés utilisant individuellement ou conjointement nos outils et la section 5 conclut tout en proposant quelques travaux futurs.

\section{Skinning dynamique}

Avant de décrire notre premier outil, nous allons tout d'abord énumérer et justifier les contraintes que nous nous sommes fixées sur la forme, la localisation et l'induction des déformations que nous souhaitons prendre en compte.

\subsection{Effets recherchés}

\subsubsection{Localisation des déformations}

Notre première observation est que l'amplitude et la fréquence de vibration des masses musculaires et graisseuses sont locales et spécifiques à chaque membre. Il est donc nécessaire d'offrir un outil qui permette de spécifier des volumes de chair indépendants ayant chacun leurs propres caractéristiques. Ainsi, ces volumes de chair peuvent être placés sur le personnage uniquement aux emplacements où ils sont nécessaires selon le type de comportement souhaité. Les articulations, principalement composées d'os et de peau, sont alors des frontières naturelles aux volumes de chair indépendants puisqu'elles ne subissent pas de déformations dynamiques.

\subsubsection{Modèle mécanique}

Les déformations d'un objet quelconque soumis à des forces ne peuvent pas se calculer de manière exacte. Il est nécessaire de discrétiser le problème en temps et en espace. Cependant, même discrétisé en éléments finis par exemple, un objet tel qu'une cuisse réelle, composée de nombreux matériaux entremêlés, mènera à un sys- 
tème d'équations complexes que nous ne pouvons résoudre en temps-réel. Afin d'effectuer un certain nombre d'approximations lors de la mise en place de notre outil, nous avons approché les déformations d'un membre réel par celles d'un modèle très simple. Cette étape de modélisation amoindrit la qualité des résultats obtenus, mais réduit considérablement les temps de calcul.

Si l'on prend l'exemple d'une cuisse, sa composition se résume à un os rigide entouré d'un volume visco-élastique que nous supposerons homogène composé d'un mélange de fibres musculaires et de tissus adipeux. Notre modèle théorique, présenté sur la figure 1, est donc constitué d'un cylindre rigide qui représente l'os entouré d'un second cylindre déformable qui modélise les muscles et les tissus. Les extrémités des deux cylindres sont des disques rigides puisqu'ils se situent au niveau des articulations.

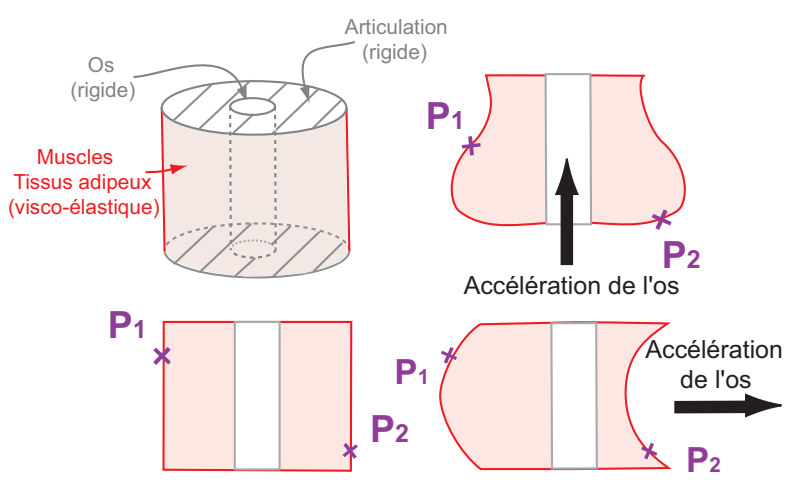

Figure 1. Un modèle mécanique simplifié d'un membre humain et ses déformations sous différentes accélérations : à gauche, le modèle, une coupe et la labellisation de deux points $P_{1}$ et $P_{2}$; à droite, accélération de l'os dans la direction de son axe (haut) et accélération de l'os dans la direction orthogonale à son axe (bas); $P_{1}$ et $P_{2}$ se trouvent déplacés dans la direction opposée à l'accélération.

Cette figure montre aussi les déformations que nous souhaitons obtenir pour différentes accélérations. En s'appuyant sur ce modèle et sur l'observation des déformations qui ont lieu lors de mouvements réels, nous proposons trois lois de comportement de la déformation.

1) Plus un point est loin de l'os, plus il est libre de se déplacer. D'autre part, le déplacement initial subi par un point s'effectue dans la direction opposée à l'accélération de l'os. On peut observer ce phénomène sur les points $P_{1}$ et $P_{2}$ de la figure 1 .

2) Les articulations doivent pouvoir bouger sans rompre la continuité qui existe entre deux éléments consécutifs. Cela nous conduit à les modéliser comme des sections rigides qui ne subissent pas de déformations dynamiques. Nous souhaitons de ce fait que les déformations soient nulles aux extrémités de l'élément (c'est à dire aux articulations) et maximales au centre de l'élément. 
3) Quel que soit le mouvement du squelette, même extrême, la chair ne doit pas pouvoir franchir l'os et se retrouver de l'autre côté. La déformation que peut subir la chair doit donc être contrainte à une déformation maximale.

\subsubsection{Rôle des os}

Dans notre approche, comme c'est le cas dans la réalité, c'est le mouvement rigide du squelette, donc des os, qui induit une déformation dynamique des masses de chair et donc de la peau, couche externe. Les os sont donc le moteur direct des déformations et offrent une information capitale quant à la forme de la déformation et son amplitude. Nous tirons donc avantage du fait que le squelette est composé d'une hiérarchie de repères et du fait que des repères supplémentaires sont présents aux bouts des membres (au bout des doigts par exemple), afin de contrôler le squelette par cinématique inverse. Leur présence a pour conséquence qu'une portion du corps d'un personnage se retrouve toujours comprise entre deux repères au minimum, ce qui va nous permettre de définir des os associés aux éléments de chair. Des repères supplémentaires peuvent être rajoutés là où l'artiste le juge nécessaire pour servir de base aux calculs de notre skinning dynamique, c'est à dire pour permettre de spécifier des $o s$ qui ont des positions et orientations adéquates.

\subsection{Principe général $d u$ skinning dynamique}

L'élément de chair dynamique permet de regrouper un ensemble de sommets du maillage en une structure qui vibre autour de l'os grâce à un ressort amorti fixé au centre de celui-ci. Le ressort permet de traîner un repère dynamique qui définit une forme dynamique de l'élément qui n'est rien d'autre que l'élément statique translaté. Chaque sommet du maillage de l'élément possède donc, à chaque instant, une position obtenue par skinning classique (point $P_{1}$ de la figure 2) et une position dynamique (point $P_{2}$ de la figure 2). La position finale de chaque sommet est obtenue par combinaison linéaire pondérée de ces deux positions. En effet, afin que la forme finale soit déformée et non une simple translation de la forme statique, il est nécessaire de spécifier des poids d'interpolation différents pour chaque sommet.

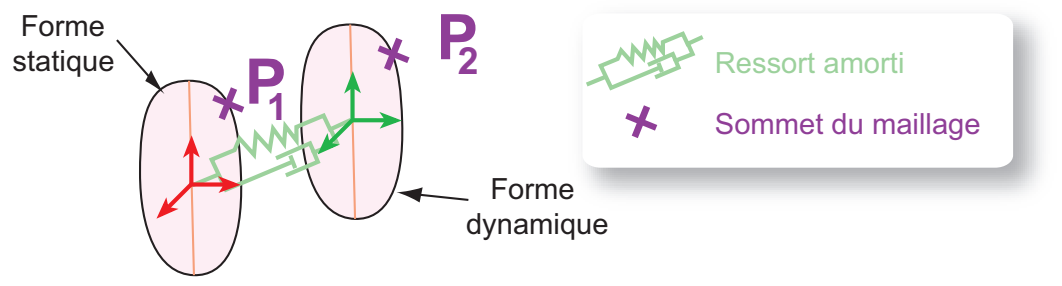

Figure 2. Deux formes clefs : la forme dynamique est la translatée de la forme statique et son mouvement est déterminé par un ressort amorti attaché à l'os. 
Lors du calcul des poids, des fonctions d'atténuation sont utilisées pour prendre en compte la localisation de la masse de chair, son volume, ainsi que la présence d'articulations, qui, essentiellement composées d'os, ne vibrent pas. Ces fonctions, ainsi que le calcul des poids, seront présentés à la section 2.5, après la description qui suit de notre méthode de spécification d'un élément de chair et de son ressort associé. Un exemple concret d'élément de chair est montré sur la figure 3.
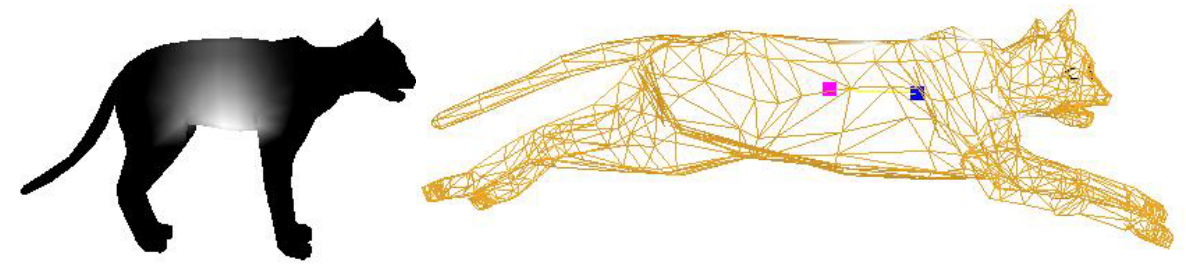

Figure 3. Un exemple concret d'élément de chair dynamique : à droite, nous pouvons voir le modèle en fil de fer ainsi que les deux repères statique (carré bleu) et dynamique (carré rose). A gauche, les poids associés calculés automatiquement variant de 0 (noir, pas de déformation) à 1 (blanc, déformation maximale).

\section{3. Élément de chair dynamique}

\subsubsection{Spécification d'un élément}

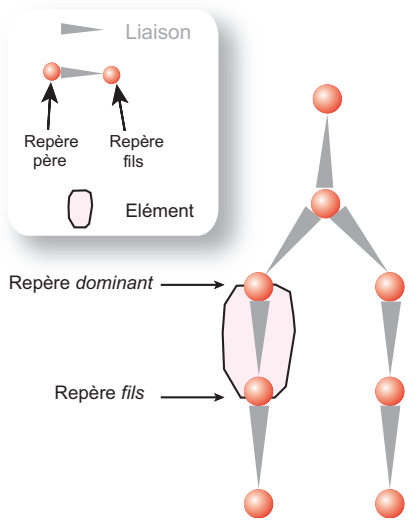

Figure 4. Un squelette d'animation et sa hiérarchie de repères contenant un élément dynamique spécifié par deux repères : le repère dominant, plus haut dans la hiérarchie, et l'un de ses repères fils. 
Un élément de chair dynamique regroupe un ensemble de sommets du maillage du modèle qui se déforment et vibrent en même temps et avec la même fréquence lorsque le repère du squelette dont ils dépendent subit un mouvement (translation ou rotation). Chaque élément dynamique est défini par l'utilisateur en sélectionnant un ensemble de sommets du maillage et au moins deux repères du squelette d'animation : un repère appelé dominant qui donne son mouvement rigide à l'élément, et un ou plusieurs repères correspondant à des fils dans la hiérarchie du squelette, qui définissent un repère moyen fils (voir figure 4). Ces deux repères définissent les extrémités de l'élément et doivent être choisis sur une zone statique du personnage qui ne subit pas de déformations dynamiques, une articulation par exemple. Ils correspondent aux deux couvercles de notre modèle mécanique (figure 1). Ces repères servent donc aussi de jonction entre deux éléments consécutifs, comme c'est le cas du bras et de l'avant bras, séparés par le coude.

Cependant, les repères choisis ne sont pas nécessairement consécutifs dans la hiérarchie. Par exemple, pour modéliser la région du ventre, bien que le squelette d'animation puisse contenir un grand nombre de vertèbres (et donc de repères dans le squelette d'animation), un seul élément de chair encadré par deux repères éloignés suffit. L'utilisateur pourra notamment prendre le bassin comme repère dominant et une vertèbre au niveau du plexus solaire comme repère fils.

Un os virtuel est alors défini par le segment de droite qui relie le repère dominant au barycentre des repères fils sélectionnés. Le milieu de l'os virtuel sera choisi comme centre du repère local de l'élément de chair, appelé $F_{s}$, et ce repère local suivra rigidement le mouvement du repère dominant au cours de l'animation. Des exemples d'éléments de chair sont montrés sur la figure 5.

\subsubsection{Repère dynamique}

Afin d'ajouter des déformations dynamiques, nous ajoutons un repère dynamique $F_{d}$ à chaque élément de façon à capturer les vibrations visco-élastiques des muscles et des tissus lors du mouvement du squelette. Ce repère est attaché au repère local $F_{s}$ par un ressort amorti de longueur à vide nulle. Lorsque le repère dominant est animé, il entraîne rigidement le repère $F_{s}$ attaché à l'os, ce qui a pour conséquence d'étirer le ressort. En plus des forces générées par le ressort, nous prenons en compte d'autres forces physiques comme une pseudo-gravité, réglable par l'utilisateur. Les équations obtenues sont résolues pendant la boucle d'animation par une intégration numérique de type Euler explicite modifié $\left(v_{t+d t}=v_{t}+\gamma_{t} d t\right.$ et $\left.u_{t+d t}=u_{t}+v_{t+d t} d t\right)$. En effet, les ressorts étant indépendants et les calculs suffisamment rapides pour prendre un petit pas de temps, n'importe quelle méthode simple d'intégration est suffisamment robuste pour ne pas diverger. On en déduit une nouvelle position de $F_{d}$ à chaque pas de l'animation. Il est important de noter que dans notre implémentation actuelle, l'orientation du repère dynamique $F_{d}$ reste la même que celle du repère $F_{s}$ pendant le mouvement. Ceci est une conséquence du fait que seule l'élongation du ressort est prise en compte dans la suite pour le calcul de la déformation. 


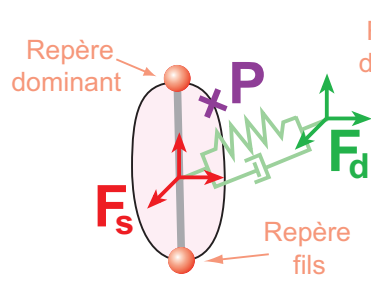

(a)

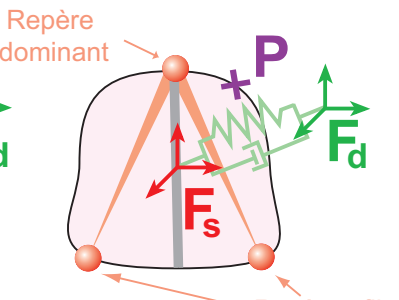

(b)

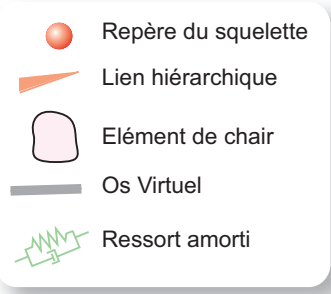

Figure 5. Un repère dynamique $F_{d}$ est connecté au repère local de l'élément de chair dynamique $F_{s}$ à l'aide d'un ressort amorti de longueur à vide nulle. (a) : le repère local se trouve au milieu de l'os virtuel délimité par un repère du squelette à chaque bout. (b) : le repère local est défini par 3 repères du squelette.

La masse ponctuelle $m$ positionnée à l'origine du repère dynamique représente la masse de l'élément de chair. La longueur à vide du ressort est prise égale à zéro pour que pendant le mouvement, le repère dynamique tende toujours à revenir vers le repère local. Ainsi, lorsque le mouvement est nul et qu' aucune force extérieure n'agit sur l'élément, les deux repères (local et dynamique) sont confondus. L'amortissement $a$ et la raideur $k$ du ressort sont des paramètres réglables par l'utilisateur, ce qui lui permet, au cours de l'animation, de régler la fréquence des vibrations et l'amplitude des déformations. Enfin, pour ne pas observer de déformations excessives, comme par exemple, la traversée de l'os d'une partie de la chair, le ressort est contraint à une élongation maximale dont le calcul sera détaillé à la section 2.6. Lorsque le ressort atteint la valeur maximale autorisée, nous corrigeons simplement la position du repère dynamique lors de l'intégration numérique en le ramenant à sa position maximale sur la direction calculée.

\subsection{Application au maillage}

À chaque pas de l'animation, les sommets du maillage du modèle sont tout d'abord déplacés par un skinning classique, en réponse au mouvement du squelette. Le skinning dynamique est ensuite appliqué sur les sommets obtenus pour calculer leur position finale. Pour chaque sommet, sa position finale est une interpolation linéaire pondérée entre la position du sommet dans le repère local de l'élément de chair et sa position dans le repère dynamique. Soit $P$ la position finale du sommet $i$. Soit $P_{1}$ la position de ce sommet dans le repère local de l'élément de chair $F_{s}$, après la première étape de skinning, soit $P_{2}$, la position de ce même point initial mais translaté par le 
ressort $^{1}$ (voir figure 2). Lorsque $P_{1}$ et $P_{2}$ sont exprimés dans le même repère $F_{s}$, la position finale $P$ se calcule de la manière suivante :

$$
P=\alpha_{1} P_{1}+\alpha_{2} P_{2}
$$

avec $\alpha_{1}$ et $\alpha_{2}$, les poids de skinning dynamique que nous devons définir. De même que dans le skinning classique, nous souhaitons assurer que $\alpha_{1}+\alpha_{2}=1$, pour que les déformations restent dans l'enveloppe convexe des positions définies par les deux repères.

On peut de plus montrer que la déformation exprimée par l'équation (1) dépend directement de l'élongation $\vec{u}$ du ressort. Soit $\vec{t}$, la translation que nous devons appliquer à $P_{1}$ pour obtenir la position finale $P: \vec{t}=P-P_{1}$. En remplaçant $P$ par son expression définie à l'équation (1), on obtient :

$$
\begin{aligned}
& \vec{t}=\left(\alpha_{1}-1\right) P_{1}+\alpha_{2} P_{2} \\
& \vec{t}=-\alpha_{2} P_{1}+\alpha_{2} P_{2} \\
& \vec{t}=\alpha_{2} \vec{P}_{1} P_{2}=\alpha_{2} \vec{u}
\end{aligned}
$$

La forme finale dépend donc uniquement du poids $\alpha_{2}$ spécifié à chaque sommet ainsi que de l'élongation et de la direction du ressort à chaque instant, exprimées par le vecteur $\vec{u}$.

\subsection{Calcul automatique des poids}

La prise en compte de la forme que l'on souhaite donner à la déformation se fait à travers les poids du skinning dynamique qui permettent de spécifier pour chaque sommet du maillage, une capacité à se déplacer. Le calcul des poids doit donc prendre en compte les trois contraintes sur la forme de la déformation présentées à la section 2.1.

Les deux premières contraintes sont modélisées par deux fonctions mathématiques qui sont combinées pour calculer l'ensemble des poids $\alpha_{2}$ associés aux sommets du maillage. Afin de pouvoir exprimer simplement nos fonctions mathématiques, nous nous plaçons dans le repère local de l'élément $F_{s}$. L'origine de ce repère se situe au milieu de l'os et l'axe des ordonnées est aligné avec l'os et dirigé vers le repère dominant. La position des deux autres axes a peu d'importance. La première fonction associe, à chaque point $P(x, y, z)$ du maillage, un réel qui ne dépend que de ses coordonnées $x, z$. La seconde fonction lui associe un coefficient compris entre 0 et 1 qui ne dépend que de la coordonnée $y$ du point.

La première contrainte prend en compte la morphologie du membre en favorisant le déplacement des points éloignés de l'os. Si l'on se réfère à l'équation (4), on re-

1. $P_{1}$ exprimé dans le repère $F_{s}$ a les mêmes coordonnées que $P_{2}$ exprimé dans le repère $F_{d}$. 
marque que plus un sommet a un poids élevé, plus il se déplace. Le poids $\alpha_{2}$ est donc directement lié à la distance du sommet à l'os. Appelons $\operatorname{shape}(x, z)$ cette fonction :

$$
\operatorname{shape}(x, z)=d(P, B)
$$

avec $P$, la position du sommet dans le repère local après skinning classique, $B$, le segment de droite représentant l'os virtuel, et $d$, la distance Euclidienne du sommet du maillage à l'os (voir figure 6 (a)).

Cependant, la fonction shape $(x, z)$ ne traduit pas forcément la réalité qui pourrait très bien mettre en jeu une fonction quadratique. Malheureusement, pour pouvoir choisir une élongation maximale pour le ressort qui garantisse à elle seule que la chair ne traversera pas l'os, $\operatorname{shape}(x, z)$ doit être inférieure à l'épaisseur de chair, pour tout $x, z$.

Afin d'accentuer les poids forts et de diminuer ou de forcer à zéro des poids plus faibles, il est possible de prendre en compte une épaisseur de l'os, soit déduite automatiquement du maillage si les os sont modélisés, soit réglée à la main par l'utilisateur. Il suffit alors de soustraire un scalaire à la distance calculée par l'équation (5) et qui correspond à l'épaisseur au point $P$.

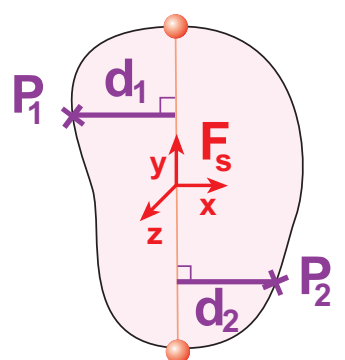

(a)

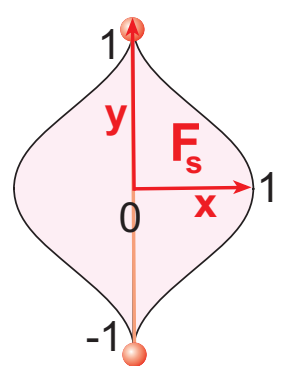

(b)

Figure 6. Le poids avant atténuation donné par la première contrainte (a) correspond à la distance Euclidienne entre le sommet considéré et l'os virtuel de l'élément. Par exemple, $P_{1}$ aura comme poids $d_{1}$ et $P_{2}, d_{2}$. La fonction d'atténuation (b) diminue les poids obtenus par shape $(x, z)(a)$ d'autant plus que sommet concerné est proche de l'une des extrémités de l'élément.

Notre seconde fonction permet de diminuer les effets dynamiques aux abords des articulations et d'assurer la continuité entre deux éléments de chair. Nous appliquons donc une atténuation des poids obtenus par shape $(x, z)$ par une valeur variant continûment de 0 aux extrémités de l'élément (pas de déformation) à 1 au centre de l'élément (déformation non atténuée).

Soit attenuation(y), la fonction mathématique qui cherche à satisfaire cette contrainte. Elle pénalise le déplacement d'un point lorsqu'il se rapproche des repères définissant l'élément de chair. Rappelons que notre repère local $F_{s}$ a été choisi pour se situer au centre de l'os. D'autre part, étant donné que nous connaissons la longueur 
de l'os, il est facile de normaliser les coordonnées $y$ de nos points du maillage afin qu'elles varient de 1 (extrémité dominante) à -1 (l'autre extrémité). Nos contraintes sur la fonction recherchée peuvent donc s'écrire de la manière suivante (voir figure 6 (b)) :

$$
\begin{aligned}
& -\operatorname{attenuation}(y)=0 \text { pour } y=-1, \\
& -\operatorname{attenuation}(y)=1 \text { pour } y=0, \\
& -\operatorname{attenuation}(y)=0 \text { pour } y=1 .
\end{aligned}
$$

La fonction mathématique continue la plus simple qui satisfasse ces contraintes est une parabole : attenuation $(y)=1-y^{2}$.

Cependant, cette fonction ne décroît pas avec une tangente nulle en -1 et 1 , ce qui se traduit par des discontinuités au niveau de la jonction entre deux éléments de chair ou bien entre un élément de chair et de la géométrie non dynamique. Ce problème est résolu par l'utilisation d'une fonction en cloche telle que la fonction polynomiale de Wyvill [WYV 86], peu coûteuse en temps de calcul : attenuation $(y)=1+$ $\frac{-4 * y^{6}+17 * y^{4}-22 * y^{2}}{9}$.

Le poids final $\alpha_{2}$ est une combinaison de shape $(x, z)$ et de $\operatorname{attenuation}(y)$. Les deux fonctions sont multipliées puis le poids obtenu est normalisé de telle sorte que les poids varient toujours de 0 à 1 :

$$
\alpha_{2}(x, y, z)=\frac{\operatorname{shape}(x, z) * \operatorname{attenuation}(y)}{\max (\text { shape } * \text { attenuation })}
$$

avec max (shape * attenuation), la valeur maximale obtenue parmi tous les sommets de l'élément de chair. Cette normalisation est nécessaire puisque des poids basés uniquement sur la distance à l'os, même avec une atténuation, dépendraient directement de l'échelle du modèle. Ceci résulterait en des déformations ou trop petites, ou complètement irréalistes. D'autre part, l'échelle du modèle est déjà prise en compte au niveau de l'élongation du ressort.

Les poids $\alpha_{2}$ ne sont calculés qu'une seule fois, pendant l'initialisation et ce calcul est immédiat. Cependant, l'utilisateur peut, au cours de l'animation, changer l'épaisseur de l'os ou la fonction attenuation auquel cas les poids sont recalculés.

\section{6. Élongation maximale du ressort}

Quel que soit le mouvement du squelette, même extrême, la chair ne doit pas pouvoir franchir l'os et se retrouver de l'autre côté. La déformation que peut subir la chair doit donc être contrainte à une déformation maximale. Par défaut, nous considérons que le seuil critique de déformation est atteint quand la chair a une épaisseur nulle à une position donnée autour de l'os. Ceci revient à spécifier, pour chaque point, un déplacement maximal à atteindre. Or, comme le montre l'équation (4), la position finale d'un sommet $P$ est donnée par $\alpha_{2}(P) * \vec{u}$, c'est à dire par une combinaison de l'élongation du ressort, qui est la même pour chaque point, et le poids de ce point, 
choisi individuellement pour refléter la capacité de ce point à se déplacer. Ainsi, le moyen évident d'imposer un déplacement maximal à tous les points est d'imposer une élongation maximale au ressort.

Cependant, pour que cette élongation maximale ait un sens, elle doit être calculée automatiquement à partir des poids générés. En effet, si l'on choisit, avant la normalisation, le poids $\alpha_{2}$ le plus élevé de tous les points comme élongation maximale, on peut garantir qu'aucun des points ne se déplacera de plus de son épaisseur. En effet, le sommet ayant le poids maximal est le seul qui bénéficie d'un déplacement complet, égal à la valeur du ressort.

\section{Plis dynamiques}

Notre outil de création de plis permet d'ajouter des plis dynamiques à une animation existante, les déformations statiques du personnage étant calculées par un skinning classique. Les plis dynamiques, par opposition à statiques, sont ceux qui se créent et se déforment pendant l'animation, ces plis n'étant pas présents au repos. Ces plis peuvent être ajoutés, avant ou pendant l'animation, localement sur le personnage. Leur animation se fait alors automatiquement à partir de la position des points du maillage obtenus par le skinning ou skinning dynamique, à chaque pas de temps.

\subsection{Principe de la technique}

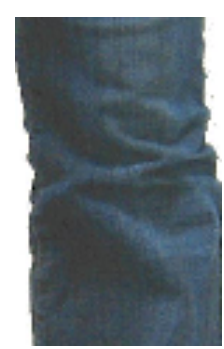

(a)

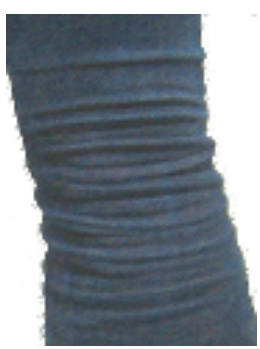

(b)

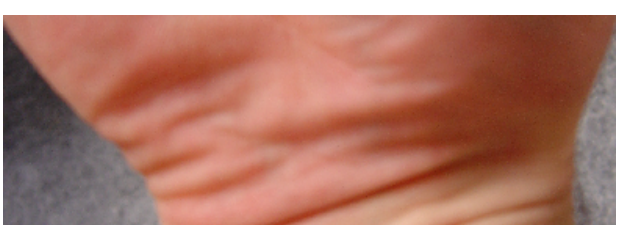

(c)

Figure 7. Quelques exemples de plis réels; Sur (c), on peut voir plusieurs rides à différents niveaux de détails.

Afin de créer un modèle adéquat, nous nous sommes tout d'abord intéressés aux origines du phénomène de plissement. Que ce soit la peau ou bien la plupart des tissus, leur principale propriété est qu'ils sont peu compressibles. C'est pourquoi, pendant le mouvement, lorsque deux parties voisines de la peau ou d'un même vêtement se rapprochent, des plis se créent pour maintenir une surface constante. 
Ces plis peuvent se créer de différentes façons. Cependant, dans tous les cas, les plis apparaissent dans une zone de compression délimitée par deux zones d'application de forces ou bien une zone d'application de force et une zone non déformée. Le lieu d'apparition des rides peut donc être encadré par deux zones extrémités qui marquent l'absence de plis (voir figure 8). Matérialisée par une double flèche sur la figure 8, la zone de compression définit la direction des plis qui lui sont orthogonaux. Dans les cas simples, les plis formés sont de la même taille, espacés régulièrement et parallèles entre eux.
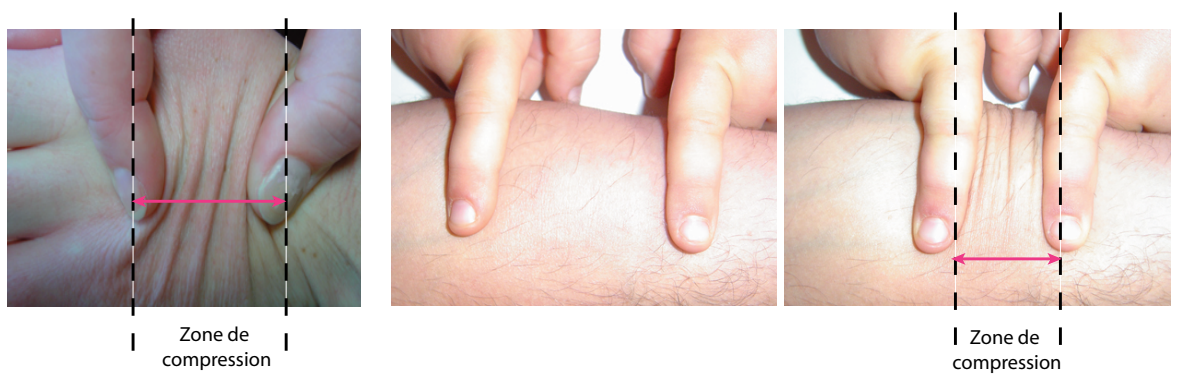

Figure 8. Apparition de plis simultanément tout le long de la zone de compression. Les plis se forment orthogonalement à la direction de compression.

Par ailleurs, la hauteur d'un pli pris individuellement n'est pas constante sur toute sa longueur. Elle est maximale au centre alors qu'elle s'atténue jusqu'à devenir nulle à chaque extrémité. La largeur des plis, et donc leur atténuation plus ou moins régulière dépend de la largeur de la zone de compression.

Enfin, si l'on observe des rides réelles comme celle de la figure 7 (c), on remarque qu'elles sont souvent composées de rides à plusieurs échelles. Ce phénomène s'observe surtout sur la peau qui n'est pas un milieu homogène, la taille des plis de vêtements étant plus directement liée à l'épaisseur et à la rigidité du tissu. Notre outil doit être capable de simuler tous ces comportements.

Le principe de notre technique est basé sur le fait que les rides ne se forment pas individuellement, et que dans chaque série de rides, l'orientation et la taille de chaque ride est similaire ainsi que l'espacement entre deux rides. D'autre part, si l'on fait des coupes transversales d'une série de rides, on peut remarquer que le profil est le même, quelle que soit la coupe. Seule l'amplitude des rides change : elle diminue au fur et à mesure que la coupe effectuée se rapproche des bords de la zone. Ce profil décrit la forme que prendrait un fil incompressible qui serait posé sur la zone de compression et attaché aux extrémités qui se rapprochent. Finalement, la seule donnée de la forme de ce fil peut être extrapolée pour obtenir la forme de tous les plis.

Nous avons donc décomposé notre algorithme dédié à la création de rides sur une surface $3 D$ en deux étapes bien distinctes. La première consiste à créer une courbe se déformant dans un plan $2 D$, servant de profil de rides et la seconde consiste à ap- 
pliquer cette courbe profil sur le maillage $3 D$. Ce profil de ride, que nous appelons courbe de contrôle pour sa capacité à contrôler les déformations $3 D$ du maillage, a pour propriété de pouvoir se déformer à longueur constante lorsque ses deux extrémités se rapprochent. Afin de garder une longueur constante, la courbe se voit contrainte à faire des plis et ce sont ces plis que nous propageons au maillage. La section suivante décrit tout d'abord un modèle de courbe de contrôle très simple puis les paramètres modifiables qui permettent d'obtenir différents comportements de cette courbe en réaction à la compression. Elle est suivie d'une section nous montrant comment cette courbe peut contrôler la déformation du maillage sur lequel elle est appliquée.

\subsection{Déformation de la courbe de contrôle}

Les rides sont animées à l'aide d'une courbe de contrôle $2 D$ discrète qui se déforme à longueur constante dans un plan orthogonal à la surface (voir figure 13). Elle est définie par ses deux extrémités, $A$ et $B$, qui sont rigidement attachées à deux sommets du maillage. La longueur de la courbe, $l$, est égale à la distance entre les deux extrémités à l'instant où elle est définie. À cet instant, le maillage n'est pas forcément au repos, la longueur $l$ de la courbe devant correspondre à son extension maximale, c'est à dire, au moment où les plis apparaissent et disparaissent. Ainsi, lorsque le maillage se déforme par skinning, les deux extrémités $A$ et $B$ se rapprochent ou s'éloignent et induisent une déformation de la courbe qui est contrainte à garder sa longueur constante. On peut assimiler cette courbe à un élastique linéaire de longueur au repos $l$ qui s'étire lorsque $A$ et $B$ s'éloignent et qui plisse lorsque $A$ et $B$ se rapprochent.

\subsubsection{Courbe de contrôle}

Au repos, la courbe est un simple segment de droite de longueur $l$ qui ne comporte aucun pli. Afin de lui permettre de se déformer, nous l'échantillonnons en $n$ points de contrôle, répartis uniformément (voir courbe du haut sur la figure 9). Ces points de contrôle servent à définir la largeur et l'espacement des plis. La distance entre deux points consécutifs est appelé le pas de la courbe.

Nous allons illustrer le principe de l'apparition des bosses à l'aide du schéma de propagation présenté sur la figure 9 , qui consiste à traiter les extrémités $A$ et $B$ différemment. L'extrémité $B$ est fixe tandis que $A$ se rapproche de $B$. Une première ride apparaît d'abord auprès de $A$, puis grandit, et enfin se propage quand elle atteint une certaine hauteur seuil. Les positions des points de contrôle de la courbe doivent donc être recalculés de façon à créer ces bosses au sein du profil de rides. Bien qu'il existe différentes stratégies de création de bosses, l'algorithme de déformation reste le même.

\subsubsection{Algorithme de déformation}

La déformation de la courbe est recalculée à chaque pas de l'animation par l'algorithme suivant : 


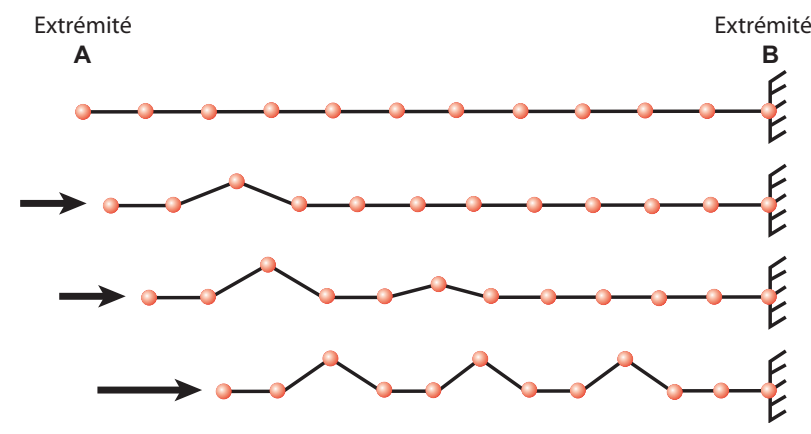

Figure 9. Une courbe de contrôle et ses déformations quand l'extrémité A se rapproche de l'extrémité B, fixe. Les bosses apparaissent d'abord auprès de A puis se propagent.

1) La nouvelle longueur du segment $\overrightarrow{A B}$ est calculée. On en déduit une nouvelle valeur du pas : pas $=\frac{\|\overrightarrow{A B}\|}{n-1}$.

2) Les points de contrôle sont réespacés sur le segment $[A B]$ suivant la valeur de pas. Ils restent donc également répartis sur le segment.

3) Certains des points de contrôle sont déplacés dans la direction orthogonale à $\overrightarrow{A B}$ et vers le haut afin de préserver la longueur totale de la courbe.

Le fait de répartir les points de contrôle uniformément sur le segment $[A B]$ permet d'assurer que chacun des plis générés du même niveau de détail aura la même largeur et de conserver, de façon exacte, la longueur de la courbe de contrôle. Les points de contrôle qui se déplacent sont choisis en fonction du schéma de plis sélectionné par l'utilisateur. Cependant, afin qu'il soit possible de superposer plusieurs séries de rides, il est impératif que les extrémités $A$ et $B$ ne soient jamais modifiées par leur propre schéma de ride. Elles ne sont donc pas déplacées.

\subsubsection{Conservation de la longueur}

Afin de préciser et d'exprimer le principe de la conservation de longueur, nous introduisons un système de coordonnées $2 D$ (nous verrons comment passer en $3 D$ à la section suivante). Soit l'extrémité $A$, l'origine du repère. $\overrightarrow{A B}$ nous donne la direction de l'axe des abscisses. L'axe des ordonnées est pris dans la direction orthogonale (voir figure 10).

Pour conserver la longueur totale, l'idée est de réinjecter, en déplaçant certains points d'une certaine hauteur sur l'axe des $y$, la longueur perdue horizontalement sur $x$ lorsque $A$ et $B$ se rapprochent. Soit $d$ le rétrécissement du segment $[A B]$ par rapport à sa position de repos $(d=\|\overrightarrow{A B}\|-l)$; soit pas, la distance entre deux points de contrôle sur l'axe des abscisses; et soit $h$, la hauteur que doit prendre le premier point de contrôle à être déplacé verticalement pour conserver la longueur totale de la courbe. 


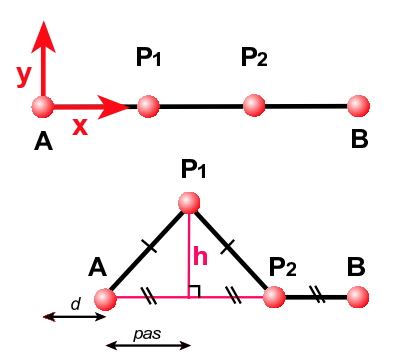

Figure 10. Le rétrécissement horizontal d est réinjecté en déplaçant $P_{1}$ dans la direction verticale, d'une hauteur $h$.

Si l'on se réfère au schéma 10 qui représente la première bosse qui se forme sur la courbe de contrôle, $A, B$ et $P_{2}$ sont des points de contrôle fixes, alors que $P 1$ sera déplacé verticalement pour absorber la perte de longueur $d$. On veut alors s'assurer que $A P_{1}+P_{1} P_{2}=A P_{2}+d$. Étant donné que les points sont également espacés, on peut facilement calculer $h$, la hauteur de $P_{1}$, en utilisant le théorème de Pythagore. On obtient :

$$
h=\sqrt{d * p a s+\frac{d^{2}}{4}}
$$

\subsubsection{Différentes stratégies possibles}

Dans la pratique, la perte de longueur $d$ est absorbée par plusieurs points de contrôle, ce qui peut se faire de plusieurs manières différentes. Nous proposons trois schémas principaux de propagation des bosses d'après nos observations : les bosses peuvent apparaître itérativement à partir de l'extrémité $A$ du segment, symétriquement à partir des deux extrémités ou bien de manière simultanée tout le long de la courbe. Dans les deux premiers cas, l'utilisateur spécifie une hauteur de bosse maximale $h_{\max }$. Lorsque cette hauteur est atteinte pour une bosse, les bosses se propagent. Dans le troisième cas, la longueur perdue $d$ est divisée par le nombre de bosses et répartie équitablement entre toutes les bosses qui grandissent alors en même temps.

La forme de la courbe peut aussi être modifiée de façon très simple en changeant quelques paramètres comme l'espacement entre les bosses (zéro ou plusieurs pas au lieu d'un seul) ou bien la largeur des bosses. En effet, une crête peut être composée d'un ou plusieurs points de contrôle. Différentes formes de courbes de contrôle sont montrées sur la figure 11 .

\subsubsection{Niveaux de détails}

Comme nous l'avons mentionné à la section 3.1, nous souhaitons pouvoir avoir des rides comportant plusieurs résolutions. Cette propriété doit être prise en compte au niveau de la courbe de contrôle. Pour cela, seulement un certain pourcentage de la longueur $d$ est réinjecté dans la courbe au niveau grossier, le reste étant gardé pour les ni- 


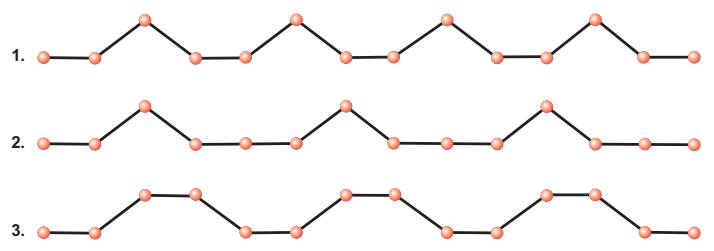

Figure 11. Différents schémas de courbes de contrôle : 1.-l'espacement entre les bosses est de 1 segment, la crête de chaque bosse est composée d'un seul point de contrôle; 2.-l'espacement entre les bosses est de 2 segments; 3.-la crête de chaque bosse est composée de 2 points de contrôle.

veaux plus fins. Naturellement, pour obtenir des rides plus fines, la courbe de contrôle doit être subdivisée. Nous doublons simplement le nombre de points de contrôle à chaque raffinement, les nouveaux points se trouvant chacun au milieu de deux points de contrôle consécutifs. La figure 12 nous montre, en (a), la courbe de contrôle subdivisée, et, en (b), le résultat de son application sur un maillage simple. L'utilisateur peut donc spécifier le nombre de niveaux de détails souhaité, de nouveaux seuils pour la hauteur des rides, ainsi que le pourcentage de la longueur qu'il souhaite retrouver à tel ou tel niveau.

Il est important de remarquer que la spécification de la taille et du niveau de détail des rides est réalisée indépendamment de la résolution du maillage sur lequel elles vont être appliquées. Au moment de la déformation du maillage, une subdivision locale détaillée à la section 3.3.5 est effectuée si nécessaire.

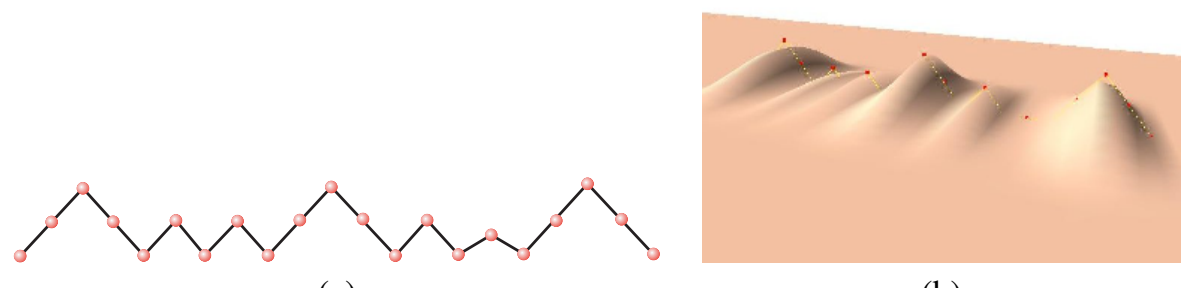

(a)

(b)

Figure 12. Rides à différents niveaux de détails : (a)-une courbe de contrôle subdivisée ; (b)-application de cette courbe sur un maillage.

\subsection{Déformation du maillage}

Cette section décrit l'utilisation de la courbe de contrôle décrite précédemment pour déformer un maillage polygonal afin de créer la géométrie $3 D$ des plis. 


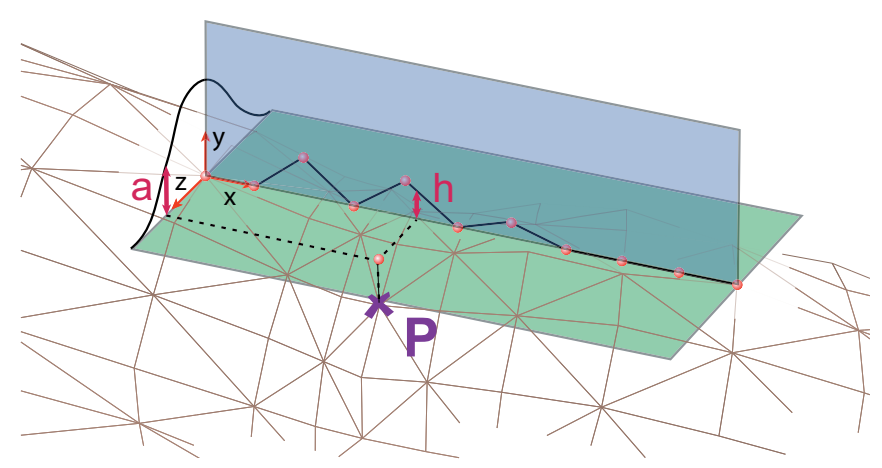

Figure 13. Calcul de l'élévation d'un sommet influencé : l'abscisse de la projection du point $P$ dans le rectangle d'influence donne la hauteur $h$ qui est atténuée par a calculée à partir de son ordonnée.

\subsubsection{Mise en place de l'outil}

La courbe de contrôle contrôle directement la déformation du maillage. Pour qu'il se mette à faire des plis lorsqu'il se retrouve compressé dans une direction, il faut que la courbe de contrôle suive les déformations du maillage induites par le skinning. C'est pourquoi elle est ancrée à ce dernier au niveau de ses extrémités $A$ et $B$.

L'infographiste, pour spécifier une série de rides, doit dessiner sur le maillage, à l'aide de la souris, un segment de droite qui correspond à la courbe de contrôle au repos. Les extrémités du segment sont attachées aux deux sommets les plus proches du maillage au sens de la distance euclidienne, l'extrémité $A$ étant le premier point spécifié par l'infographiste, $B$ le second. La direction du segment détermine la direction des rides qui lui seront perpendiculaires, tandis que sa longueur nous donne la longueur au repos de la courbe, $l$.

Ensuite, l'infographiste peut régler les paramètres de la courbe comme le choix de la stratégie de propagation, l'espacement des rides, leur hauteur, et les niveaux de détails. Tous ces paramètres peuvent ensuite être modifiés interactivement, aussi bien pendant la phase de modélisation que pendant la phase d'animation.

Lors de l'animation, la courbe de contrôle se déforme dans le plan défini par la direction du vecteur $\overrightarrow{A B}$, désigné dans la suite par l'abscisse $x$, et la direction moyenne des deux normales à la surface aux extrémités $A$ et $B$, désignée par l'ordonnée $y$ dans la suite (voir figure 13). Le plan $(x, y)$ dans lequel la courbe se déforme, la position des extrémités $A$ et $B$ de la courbe et donc sa longueur courante sont réévalués avant chaque rendu pour permettre à la courbe de contrôle de se déformer. 


\subsubsection{Région d'influence}

Comme nous l'avons vu à la section 3.1, les plis apparaissent uniquement dans la zone de compression. Il va donc falloir préciser l'étendue de cette zone. Les sommets du maillage qui s'y trouvent seront alors influencés par la courbe de contrôle. Associée à chaque courbe, nous définissons une région d'influence qui s'étend de part et d'autre de la courbe. Concrètement, cela se présente sous la forme d'un rectangle centré sur le segment $[A B]$ dont la largeur est réglable par l'animateur. On peut voir une telle région d'influence sur la figure 13.

Les sommets du maillage qui seront influencés par l'outil et donc déplacés sont ceux qui se projettent dans le rectangle d'influence de l'outil, la projection se faisant selon la normale au plan du rectangle, et parmi eux, ceux dont le produit vectoriel de la normale par celle du rectangle d'influence est positif.

\subsubsection{Atténuation aux bords de la région d'influence}

L'utilisateur peut donc, en jouant sur la largeur du rectangle, définir la longueur des plis. En plus de ce paramètre, il peut choisir un profil d'atténuation des plis qui décrit comment leur hauteur décroît au fur et à mesure que l'on s'éloigne (sur $z$ ) de la courbe de contrôle, c'est à dire le long d'une ride (voir figure 13). Le coefficient d'atténuation est toujours compris entre 1 , au centre du rectangle, et 0 , aux bords. Dans notre implémentation, l'utilisateur peut choisir de ne pas mettre d'atténuation, ce qui n'est pas très réaliste, ou bien, s'il en met, entre deux schémas d'atténuation différents.

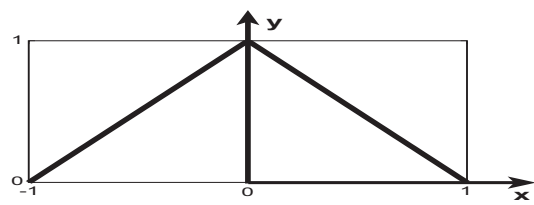

(a)

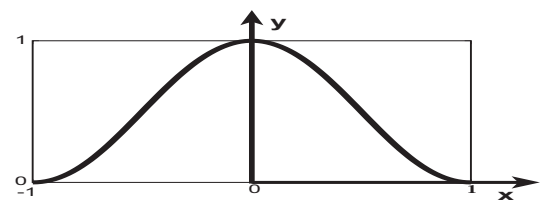

(b)

Figure 14. Un profil d'atténuation définit comment les bosses décroissent jusqu'à s'annuler aux bords du rectangle d'influence; (a) $y=1-a b s(x)$; (b) $y=$ $1+\frac{\left(-4 x^{6}+17 x^{4}-22 x^{2}\right)}{9}$.

Le premier schéma d'atténuation, représenté sur la figure 14, est linéaire ( $y=$ $1-a b s(x))$ et très rapide à calculer mais n'a pas de tangentes nulles en $-1,0$ et 1 . Cela se traduit par des discontinuités aux bords du rectangle, c'est-à-dire entre la zone des plis et les zones sans pli.

Le second utilise la fonction polynomiale de Wyvill [WYV 86], en forme de cloche $\left(y=1+\frac{\left(-4 x^{6}+17 x^{4}-22 x^{2}\right)}{9}\right)$. Cette fonction nous apporte les avantages d'une gaussienne (forme lisse, en cloche), a des tangentes nulles en $-1,0$, et 1 , et est rapide à résoudre pour un $x$ donné. En effet, la rapidité de résolution de l'équation est une 
donnée très importante à prendre en compte puisque le coefficient d'atténuation de chaque point de la zone d'influence doit être recalculé à chaque pas de temps.

\subsubsection{Déplacement des sommets influencés}

Pendant l'animation du personnage, le squelette entraîne une déformation statique de la peau qui lui est attachée (skinning), et éventuellement des déformations dynamiques simulant la vibration de la chair (skinning dynamique). Le maillage est donc déformé en réponse au mouvement du personnage, et les sommets $A$ et $B$ de la courbe de contrôle sont déplacés. Pour chaque sommet du maillage influencé par l'outil, on calcule alors le déplacement qu'il doit subir. Il est obtenu par les coordonnées $(x, z)$ de sa projection sur le rectangle d'influence selon la normale de celui-ci. La coordonnée $x$ donne la hauteur $h$ du point sur la courbe de contrôle, et la coordonnée $y$ décrit l'atténuation $a$ subie par ce point au fur et à mesure qu'il s'éloigne du centre du rectangle. Le schéma 13 nous montre l'obtention de $h$ et $a$ pour un point $P$ quelconque.

Concrètement, l'élévation finale du point est sa hauteur $h$, multipliée par l'atténuation $a$ : elevation $=h * a$. Cette élévation est appliquée au point, dans la direction de sa normale à la surface avant application de la ride.

\subsubsection{Subdivision du maillage}

Pour obtenir de bons résultats, cette méthode nécessite un maillage suffisamment échantillonné par rapport à la courbe de contrôle pour que les rides puissent s'exprimer. Demander à l'infographiste de raffiner à la main le maillage dans les zones où il place les rides serait complètement à l'encontre du principe de la technique qui se veut simple et non pénible à utiliser. En effet, cela forcerait l'animateur à prévoir l'emplacement des plis potentiels au préalable, ce qui serait largement aussi pénible que de les modéliser à la main, à l'aide de formes clefs. L'autre inconvénient majeur serait que l'animation en elle-même se trouverait ralentie à cause d'un nombre de polygones élevé, aussi bien au niveau du skinning, de l'ajout des effets dynamiques ou du traitement des collisions. Enfin, cela forcerait aussi à utiliser un maillage fin pour le rendu, et ceci même lorsque les rides ne seraient pas visibles (cas où la courbe de contrôle n'est pas compressée à ce moment de l'animation par exemple).

Nous avons donc choisi de raffiner le maillage localement, uniquement lorsque ceci est nécessaire. Ainsi, lorsque la zone des rides est en étirement, le maillage n'est pas subdivisé. D'autre part, lorsqu'un triangle est recouvert par plusieurs rides, nous subdivisons au niveau le plus fin nécessaire. L'algorithme de subdivision que nous utilisons est un butterfly modifié [DYN 90, ZOR 96]. En effet, cet algorithme a l'avantage d'être interpolant, ce qui nous évite de recalculer un certain nombre de propriétés des points déjà existants telles que la position, la normale, ..., mais est aussi applicable directement sur un maillage triangulaire.

Il faut aussi remarquer que c'est l'étape de subdivision, effectuée à chaque rendu, qui est la partie coûteuse de l'algorithme, tout simplement car la recherche du voisinage des triangles n'est pas trivial. Nous gardons du temps-réel, voire interactif, car 
le nombre d'étapes de subdivision est généralement petit (1 ou 2, sachant qu'à chaque subdivision, la taille des triangles est divisée par 4). Un bon espoir pour la réduction des temps de calcul est la naissance de la programmation d'algorithmes de subdivision sur les cartes graphiques [BOL 04].

\section{Résultats et performances}

Les résultats ${ }^{2}$ présentés dans cette section ont été réalisés en temps-réel sur un ordinateur standard équipé d'un processeur AMD Athlon XP 1700+, d'une RAM de 1 Giga et d'une carte graphique récente. Avant toute chose, il convient de préciser que les résultats d'un algorithme ajoutant des effets dynamiques à une animation sont très difficiles à apprécier à travers des images fixes. Il serait plus approprié de se référer aux vidéos. Néanmoins, l'effet de notre outil est représentable par le champ de déplacement des sommets, entre leur position issue du skinning classique et leur position issue du skinning dynamique. Nous avons représenté ce champ par des petits traits (bleus) sur les différentes figures.

\subsection{Skinning dynamique : un cas simple}

Notre skinning dynamique a été testé avec succès sur un modèle très simple de champignon. Le squelette est constitué de deux repères : la racine au niveau du chapeau du champignon et son repère fils situé à la base du pied. Nous avons créé un seul élément de chair, englobant tout le maillage et dépendant de ces deux repères. Le ressort responsable des mouvements dynamiques est donc situé au milieu du pied du champignon et provoque des vibrations lorsque le modèle est en mouvement (voir figure 15).

\subsection{Skinning dynamique : mouvement rapide sur un animal}

Nous avons souhaité reproduire les ballottements du ventre du chat lorsque celuici court puis saute. Pour cela, bien que le dos du chat soit constitué d'un grand nombre de vertèbres, un seul élément de chair a été utilisé. Il englobe le maillage compris entre la première vertèbre après le bassin et la dernière vertèbre avant le cou. Le ressort se retrouve donc attaché approximativement au milieu du dos de l'animal (voir figure 16).

\subsection{Déformations cinématiques : rides du front}

Nous avons appliqué notre outil de création de plis sur les fronts de deux modèles de tête différents. Étant donné que nous n'avions pas de logiciel d'animation faciale à

2. Les vidéos correspondantes sont disponibles à l'URL suivante : http ://wwwevasion.imag.fr/Publications/2006/LCA06/. 

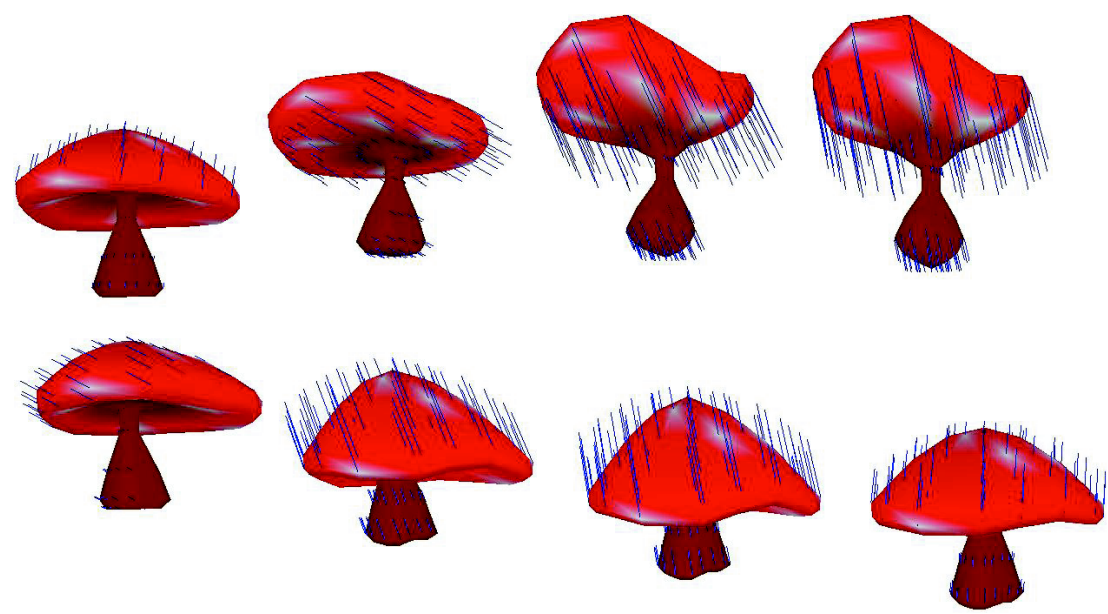

Figure 15. Un modèle de champignon surfacique déformé par notre méthode de skinning dynamique. Le mouvement est induit par un squelette rigide placé au milieu du modèle et qui est défini par deux repères : le repère dominant au niveau du chapeau du champignon et son repère fils à la base du pied.

notre disposition, nous avons manuellement changé la longueur au repos de la courbe de contrôle pour simuler une compression du maillage. Les résultats obtenus, pour différents paramètres de la courbe de contrôle, sont montrés sur la figure 17.

\subsection{Déformations cinématiques : plis au niveau des articulations}

La principale cible de notre outil de création de plis est la modélisation des plis qui se créent au niveau des articulations lorsque celles-ci plient. La figure 18 montre les résultats que l'on obtient sur l'animation d'un poignet. La rangée du haut montre les déformations dues au skinning seul, alors que la rangée du bas nous montre l'effet de notre outil. Sur ce modèle composé de 2480 polygones, nous avons utilisé une seule courbe de contrôle. L'animation se déroule à 23 frames par seconde lorsque le maillage se subdivise à deux niveaux, et à 188 frames par seconde lorsque le maillage n'est pas subdivisé, c'est à dire quand la courbe de contrôle n'est pas active.

\subsection{Déformations cinématiques : plis de vêtements}

Des formes de plis plus compliquées et variées peuvent être obtenues en combinant plusieurs séries de rides. Pour cela, on peut superposer différents outils. C'est par exemple le genre de rides que l'on observe sur les pantalons au niveau du genou. Dans ce cas, l'utilisateur dessine plusieurs profils de rides, qui se croisent. Lors du 

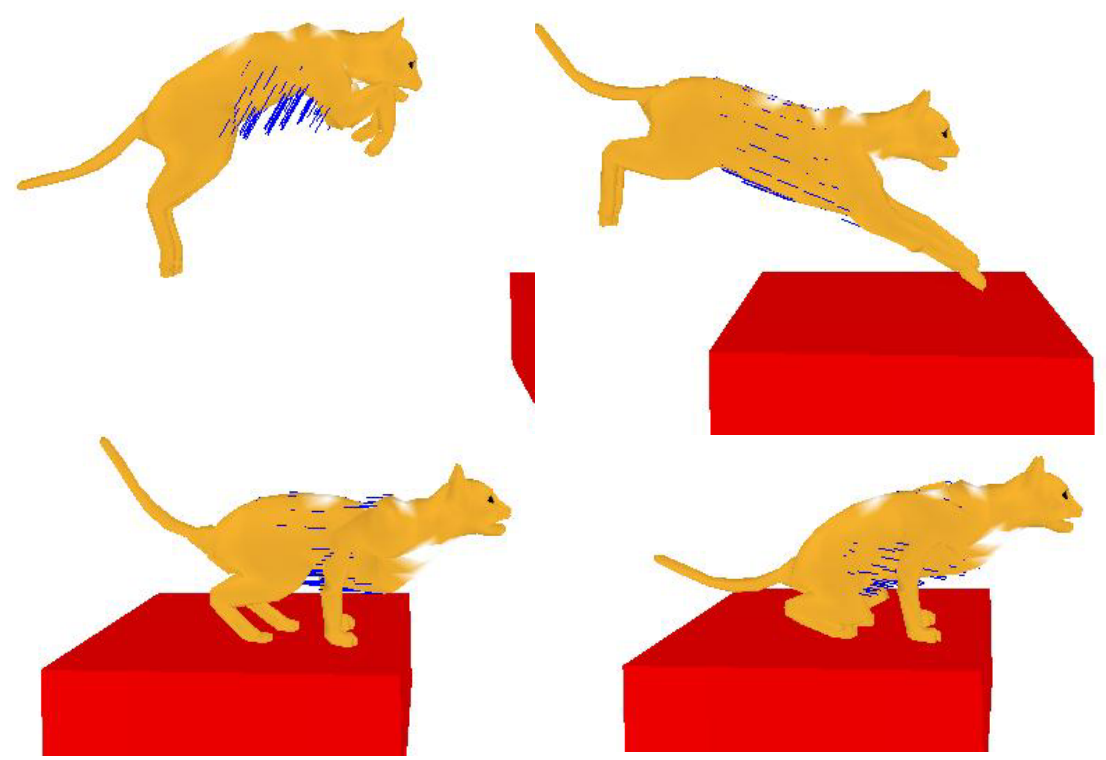

Figure 16. Le même modèle de chat, déformé en utilisant le même élément de chair dans la région du ventre, dans un mouvement de saut.

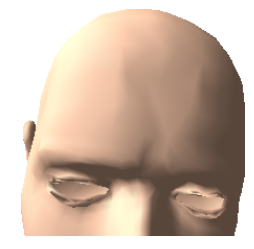

(a)

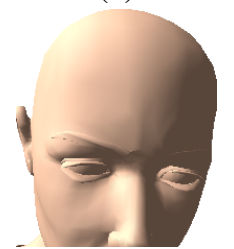

(d)

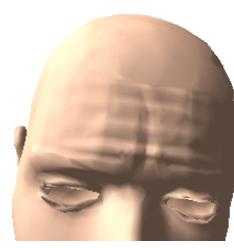

(b)

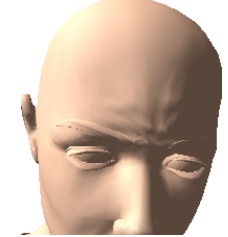

(e)

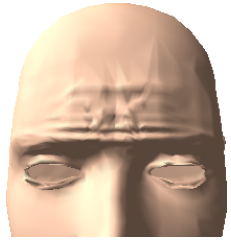

(c)

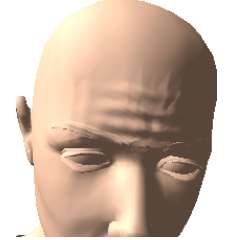

(f)

Figure 17. Utilisation de notre outil pour créer des rides sur les fronts de deux modèles différents : un modèle d'homme pour la rangée du haut et de femme pour la rangée du bas. (a) et (d)-maillages non déformés; $(b),(c),(e)$ et $(f)$-maillages déformés avec différents paramètres. 


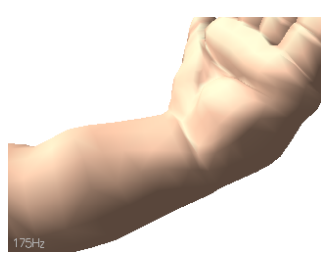

(a)

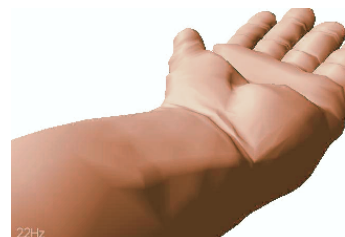

(b)

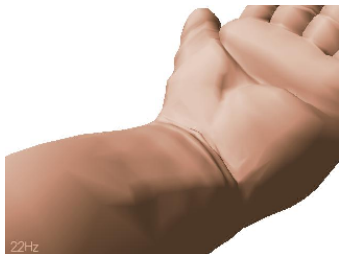

(c)

Figure 18. Illustration de l'utilisation de notre outil sur un poignet de bébé. (a)animation standard n'utilisant que le skinning classique : pas de rides présentes; (b) et (c)-notre outil fait apparaître des plis dans le creux du poignet pendant le mouvement.

calcul des déplacements des sommets influencés, l'élévation imposée aux sommets est la somme des élévations provoquées par chaque profil de plis. La figure 19 illustre l'utilisation de notre outil sur un pantalon. Nous avons placé 4 courbes de contrôle sur notre modèle, deux sur l'avant du genou (rangée du bas), et deux qui se recouvrent sur l'arrière (rangée du haut). L'animation tourne à 17 frames par seconde lorsqu'un niveau de subdivision est actif pour un maillage composé de 6362 polygones. Le frame rate descend malheureusement à 7.5 frames par seconde lorsque l'on modélise des plis plus fins qui nécessitent deux niveaux de subdivision.

\subsection{Combinaison des deux outils}

Chacun de nos deux outils agit sur le maillage polygonal qui est donné en entrée (résultant du skinning classique) et produit un maillage polygonal déformé, pouvant contenir plus de primitives dans le cas des plis. L'utilisation de ces deux outils sous forme de couches est donc tout à fait naturelle. Néanmoins, bien que les deux outils soient empilables de façon quelconque, il est préférable d'utiliser d'abord le skinning dynamique. En effet, les effets du skinning dynamique sont plus grossiers d'une part, et la génération de triangles supplémentaires par l'outil de création de plis ne peut que ralentir le skinning dynamique si celui-ci intervient en second lieu d'autre part.

Ainsi, notre dernier exemple (voir figure 20) combine notre outil de création de plis avec notre skinning dynamique permettant de simuler les effets d'inertie des masses musculaires et graisseuses. Ainsi, le ventre du chat réagit au mouvement du squelette en ballottant alors que des plis se forment autour des pattes avant et des pattes arrières. L'animation originale, par skinning simple, tourne en temps-réel (limité à 25 frames par secondes). L'animation améliorée par l'ajout de dynamique et de plis s'éxécute aussi en temps-réel. Nous n'avons malheureusement pas de statistiques plus précises, ni utilisé nos outils sur de plus gros modèles. Le modèle du chat contient 2574 polygones et l'animation présentée utilise un élément de chair et deux séries de plis. 

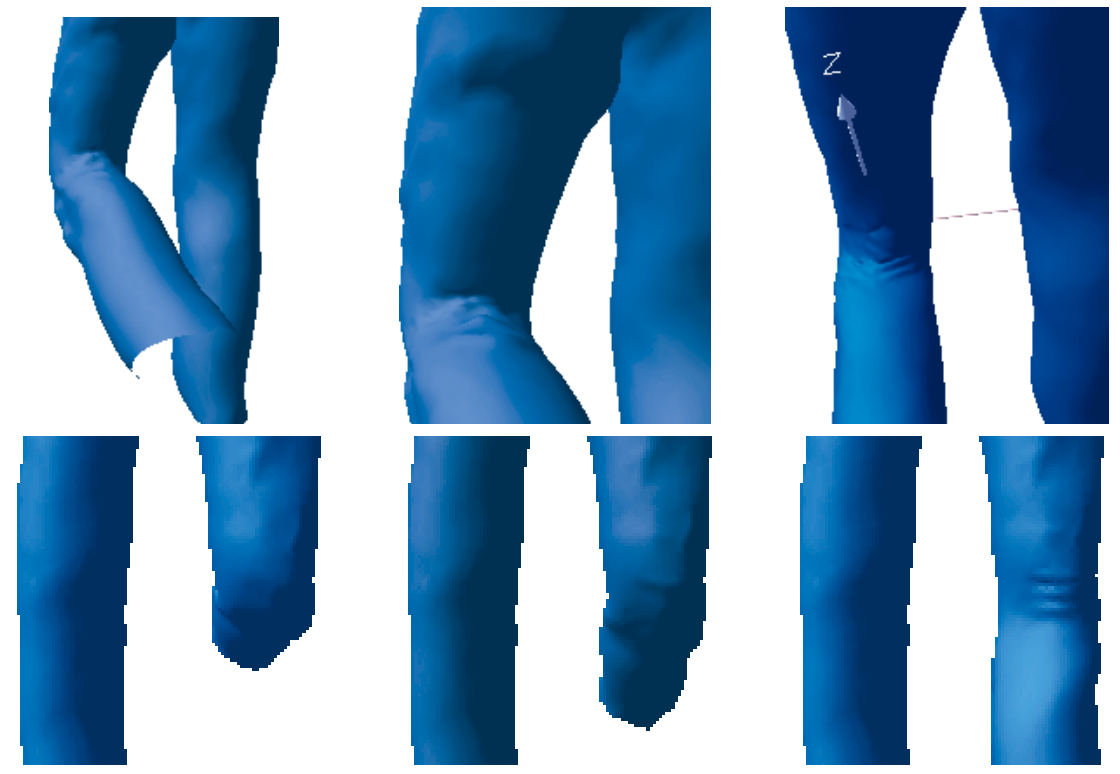

Figure 19. Illustration de notre outil de création de plis sur un pantalon virtuel. La ligne du haut montre l'arrière d'un pantalon où les plis sont créés par deux séries de plis superposées. La ligne du bas montre l'avant d'un pantalon où une série de rides est disposée sur la jambe de droite. On peut constater la différence avec la jambe de gauche, dépourvue de plis.

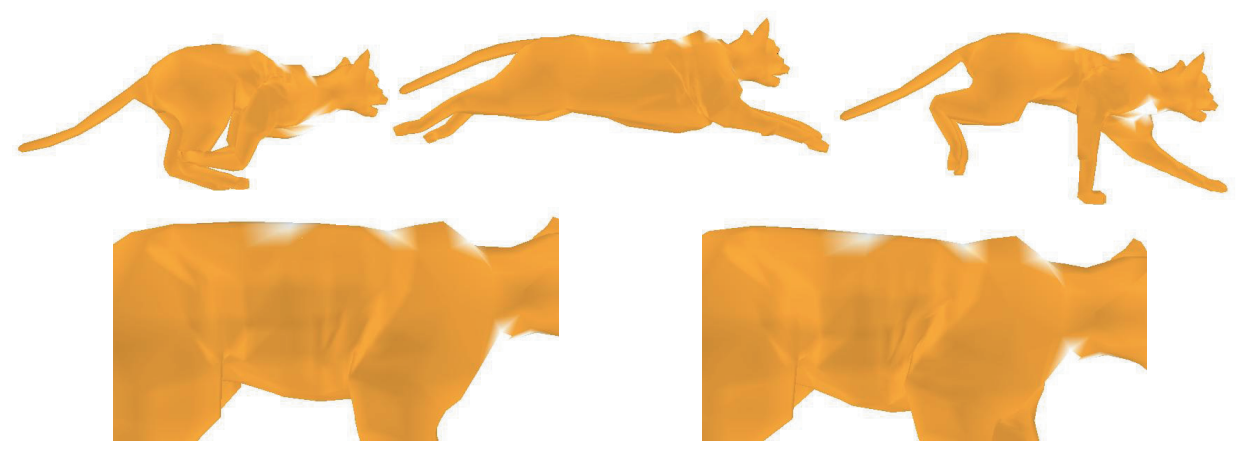

Figure 20. Animation du modèle du chat sur laquelle des effets dynamiques et des plis dynamiques ont été ajoutés.

\section{Conclusion et travaux futurs}

Nous avons proposé, dans cet article, deux techniques temps-réel permettant d'ajouter des détails à une animation de personnage. La représentation par maillage surfa- 
cique et l'animation par skinning étant des procédés standards dans le monde de l'animation $3 D$, nous avons élaboré nos techniques afin qu'elles se greffent directement sur l'existant.

Notre skinning dynamique utilise un modèle physique très simple qui, associé à un ensemble de poids, permet d'ajouter des déformations dynamiques aux parties charnues d'un personnage dont les déformations cinématiques ont été calculées par skinning. L'utilisateur peut contrôler les déformations à plusieurs niveaux grâce à des paramètres intuitifs : directement au niveau du ressort en jouant avec les paramètres élastiques de ce dernier ainsi que sur la masse de la zone de chair représentée par l'élément dynamique, et au niveau du calcul des poids de skinning, par l'intermédiaire de fonctions.

D'autre part, nous avons présenté un outil géométrique permettant de créer des plis en temps-réel sur une surface polygonale quelconque. Cette surface doit être préalablement animée par skinning et notre outil agit dans les zones où le maillage se trouve compressé en créant des plis. Plusieurs paramètres sont à la disposition de l'utilisateur afin qu'il puisse régler la fréquence des plis, leur forme, leur hauteur ainsi que leur largeur. Ces paramètres sont intuitifs, et faciles à régler, le retour visuel étant disponible en temps-réel.

Ces deux techniques agissent comme des couches indépendantes qui peuvent être rajoutée à un modèle classique et peuvent être utilisées seules ou bien combinées. Des améliorations sont envisagées pour chacune des deux techniques, que ce soit au niveau du choix des fonctions d'atténuation ou bien au niveau du fonctionnement de la courbe de contrôle des plis par exemple. Ainsi, nous aimerions pouvoir prendre en compte la rotation du ressort lors des déformations dynamiques ou bien la possibilité de créer des plis courbes.

À plus long terme, nous aimerions ajouter un système de détection de collisions et d'auto-collisions ainsi qu'une réponse adéquate de la déformation, qui sont nécessaires aussi bien au niveau du skinning dynamique pour éviter les collisions entre une partie subissant des déformations et l'autre n'en subissant pas ou bien pour les plis dans les zones très concaves.

Enfin, une des motivations principales pour la structure de nos algorithmes et notamment la subdivision à la volée en ce qui concerne les plis, est que nous souhaitons implémenter ces outils sur cartes graphiques. Les algorithmes, ne modifiant finalement que la position des sommets du maillage, pourraient être aisément implémentés dans un vertex program.

\section{Bibliographie}

[ali] « Alias-Wavefront, Maya », http ://www.aliaswavefront.com.

[BAN 02] BAndo Y., Kuratate T., Nishita T., « A Simple Method for Modeling Wrinkles on Human Skin », Proceedings of Pacific Graphics, octobre 2002.

[BAR 03] BARAFF D., Witkin A., KASS M., «Untangling Cloth », Proceedings of SIGGRAPH'03, ACM Transactions on Graphics, juillet 2003, p. 862-870. 
[BLI 78] BLINN J. F., « Simulation of wrinkled surfaces », Proceedings of SIGGRAPH'78, ACM Press, 1978, p. 286-292.

[BLO 02] Bloomenthal J., « Medial-based vertex deformation », Proceedings of the ACM SIGGRAPH Symposium on Computer Animation, ACM Press, 2002, p. 147-151.

[BOI 00] Boissieux L., Kiss G., Magnenat-Thalmann N., KalRa P., « Simulation of Skin Aging and Wrinkles with Cosmetics Insight », Computer Animation and Simulation 2000, août 2000, p. 15-27.

[BOL 04] Bolz J., Schroder P., « Evaluation of Subdivision Surfaces on Programmable Graphics Hardware », submitted for publication 2004.

[CAP 02] Capell S., Green S., Curless B., Duchamp T., Popovic Z., « Interactive Skeleton-Driven Dynamic Deformations », Proceedings of SIGGRAPH'02, ACM Transactions on Graphics, vol. 21, juillet 2002, p. 586-593.

[CHA 89] Chadwick J. E., Haumann D. R., Parent R. E., « Layered Construction for Deformable Animated Characters », Proceedings of SIGGRAPH'89, ACM Computer Graphics, vol. 23, ACM Press, 1989, p. 243-252.

[CHO 02] CHOI K., Ko H., «Stable but Responsive Cloth », Proceedings of SIGGRAPH'02, ACM Transactions on Graphics, vol. 21, $\mathrm{n}^{\circ}$ 3, 2002, p. 604-611.

[COM 02] Combaz J., Neyret F., « Painting Folds using Expansion Textures », Proceedings of Pacific Graphics'02, octobre 2002.

[DYN 90] Dyn N., Levine D., Gregory J. A., « A Butterfly Subdivision Scheme for Surface Interpolation with Tension Control », ACM Transactions on Graphics, vol. 9, $\mathrm{n}^{\circ} 2$, 1990, p. 160-169, ACM Press.

[HAD 99] Hadap S., Bangerter E., Volino P., Magnenat-Thalmann N., « Animating Wrinkles on Clothes », IEEE Visualization '99, IEEE Computer Society Press, octobre 1999, p. 175-182.

[JAM 02] JAMES D. L., PAI D. K., « DyRT : Dynamic Response Textures for Real Time Deformation Simulation with Graphics Hardware », Proceedings of SIGGRAPH'02, ACM Transactions on Graphics, vol. 21, San Antonio, TX, juillet 2002, p. 582-585.

[KON 03] Kono H., Genda E., « Wrinkle Generation Model for 3D Facial Expression », Sketches and Applications, SIGGRAPH'03, , 2003.

[KRY 02] KRY P. G., JAMES D. L., PAI D. K., « EigenSkin : real time large deformation character skinning in hardware », Proceedings of the ACM SIGGRAPH Symposium on Computer animation, ACM Press, 2002, p. 153-159.

[LAR 04a] LARboulette C., « Traitement temps-réel des déformations de la peau et des tissus sous-cutanés pour l'animation de personnages », PhD thesis, INSA Rennes, 2004.

[LAR 04b] Larboulette C., CANi M.-P., «Real-Time Dynamic Wrinkles », Proceedings of Computer Graphics International'04, IEEE Computer Society Press, juin 2004, p. 522525 .

[LEW 00] Lewis J. P., Cordner M., Fong N., « Pose Space Deformation : A Unified Approach to Shape Interpolation and Skeleton-Driven Deformation », Proceedings of SIGGRAPH'00, ACM Computer Graphics, ACM Press/Addison-Wesley Publishing Co., juillet 2000, p. 165-172.

[M“02] Müller M., Dorsey J., McMillan L., Jagnow R., Cutler B., « Stable realtime deformations », Proceedings of the ACM SIGGRAPH symposium on Computer ani- 
30 L'objet $-6 / 2006$.

mation, ACM Press, 2002, p. 49-54.

[MOH 03] Mohr A., Gleicher M., « Building efficient, accurate character skins from examples », Proceedings of SIGGRAPH'03, ACM Transactions on Graphics, vol. 22, ACM Press, 2003, p. 562-568.

[PAS 01] PASquariello S., Pelachaud C., « Greta : A Simple Facial Animation Engine », Proceedings of the 6th Online World Conference on Soft Computing in Industrial Applications, septembre 2001.

[PEN 89] Pentland A., Williams J., « Good Vibrations : Modal Dynamics for Graphics and Animation », Proceedings of SIGGRAPH'89, ACM Computer Graphics, vol. 23, ACM Press, 1989, p. 207-214.

[SAU 04] Sauvage B., Hahmann S., Bonneau G.-P., « Length Preserving Multiresolution Editing of Curves », Computing, vol. 72, 2004, p. 160-171.

[TUR 93] Turner R., Thalmann D., « The Elastic Surface Layer Model for Animated Character Construction », Proceedings of Computer Graphics International'93, , 1993, p. $399-412$.

[VIA 92] Viaud M.-L., YAHIA H., « Facial Animation with Wrinkles », Eurographics Workshop on Animation and Simulation, septembre 1992.

[VOL 99] Volino P., Magnenat-Thalmann N., « Fast Geometrical Wrinkles on Animated Surfaces », Seventh International Conference in Central Europe on Computer Graphics and Visualization (Winter School on Computer Graphics), février 1999.

[WAN 02] WANG X. C., PHILliPS C., « Multi-weight enveloping : least-squares approximation techniques for skin animation », Proceedings of the ACM SIGGRAPH symposium on Computer animation, ACM Press, 2002, p. 129-138.

[WIL 97a] WILHELms J., «Animals With Anatomy », IEEE Computer Graphics and Applications, vol. 17, $\mathrm{n}^{\circ}$ 3, 1997, p. 22-30.

[WIL 97b] Wilhelms J., Gelder A. V., « Anatomically based modeling », Proceedings of SIGGRAPH'97, ACM Computer graphics, ACM Press/Addison-Wesley Publishing Co., août 1997, p. 173-180.

[WU 94] Wu Y., Thalmann N. M., Thalmann D., « A Plastic-visco-elastic Model for Wrinkles in Facial Animation and Skin Aging », Proceedings of Pacific Graphics'94, 1994, p. 201-213.

[WU 96] Wu Y., Kalra P., Thalmann N. M., « Simulation Of Static And Dynamic Wrinkles Of Skin », Proceedings of Computer Animation '96, IEEE Computer Society Press, juin 1996, p. 90-97.

[WU 99] Wu Y., Kalra P., Moccozet L., Magnenat-Thalmann N., « Simulating wrinkles and skin aging », The Visual Computer, vol. 15, $\mathrm{n}^{\circ}$ 4, 1999, p. 183-198.

[WYV 86] Wyvill G., McPheeters C., Wyvill B., « Data Structure for Soft Objects », The Visual Computer, vol. 2, n ${ }^{\circ} 4,1986$, p. 227-234.

[ZOR 96] ZORIN D., SCHRÖDER P., SWELDENS W., «Interpolating Subdivision for meshes with arbitrary topology », Proceedings of SIGGRAPH '96, ACM Press New York, NY, USA, 1996, p. 189-192. 